\title{
A RELATIONSHIP BETWEEN RATIONAL AND MULTI-SOLITON SOLUTIONS OF THE BKP HIERARCHY
}

\author{
J. J. C. NIMMO \\ Department of Mathematics, University of Glasgow, Glasgow G12 8QQ, UK \\ e-mail: j.nimmo@maths.gla.ac.uk \\ and A. YU. ORLOV \\ Oceanology Institute, Nahimovskii Prospekt 36, Moscow, Russia \\ e-mail:orlovs@wave.sio.rssi.ru
}

(Received 22 January, 2004; accepted 18 March, 2004)

\begin{abstract}
We consider a special class of solutions of the BKP hierarchy which we call $\tau$-functions of hypergeometric type. These are series in Schur $Q$-functions over partitions, with coefficients parameterised by a function of one variable $\xi$, where the quantities $\xi(k), k \in \mathbb{Z}^{+}$, are integrals of motion of the BKP hierarchy. We show that this solution is, at the same time, a infinite soliton solution of a dual BKP hierarchy, where the variables $\xi(k)$ are now related to BKP higher times. In particular, rational solutions of the BKP hierarchy are related to (finite) multi-soliton solution of the dual BKP hierarchy. The momenta of the solitons are given by the parts of partitions in the Schur $Q$-function expansion of the $\tau$-function of hypergeometric type. We also show that the KdV and the NLS soliton $\tau$-functions coinside the BKP $\tau$-functions of hypergeometric type, evaluated at special point of BKP higher time; the variables $\xi$ (which are BKP integrals of motions) being related to KdV and NLS higher times.
\end{abstract}

2000 Mathematics Subject Classification. 35Q51, 35Q58, 05 E05.

1. Introduction. The BKP hierarchy was introduced in $[\mathbf{1}, \mathbf{2}]$ as a particular reduction of the KP hierarchy of integrable equations [1, 7]. Like the well-known KP hierarchy, the BKP hierarchy possesses multi-soliton and rational solutions. In $[3,4]$, the role of projective Schur functions ( $Q$-functions) [6] in obtaining rational solutions of the BKP hierarchy was explained. In fact, the $Q$-functions are polynomial $\tau$-function solutions of the BKP hierarchy Hirota equations and these are connected to the rational solutions through a change of dependent variables.

In [9], certain hypergeometric series in $Q$-functions (see (85) below) were shown to be $\tau$-functions of the BKP hierarchy. These $\tau$-functions are series of the form

$$
\tau\left(\mathbf{t}_{o}, \xi, \mathbf{t}_{o}^{*}\right)=\sum_{\lambda \in \mathrm{DP}} e^{\sum_{\mathrm{i}=1}^{\infty} \xi_{\lambda_{\mathrm{i}}}} Q_{\lambda}\left(\frac{1}{2} \mathbf{t}_{o}\right) Q_{\lambda}\left(\frac{1}{2} \mathbf{t}_{0}^{*}\right),
$$

where $\xi=\left\{\xi_{m}: m=1,2, \ldots\right\}$ are arbitrary parameters, $\xi_{0}=0, Q_{\lambda}$ denote projective Schur function, and the sum is over the set DP of all partitions $\lambda=\left(\lambda_{1}, \lambda_{2}, \ldots, \lambda_{k}\right)$ with distinct parts $\lambda_{1}>\lambda_{2}>\cdots>\lambda_{k} \geq 0$. Considered as a function of the variables $\mathbf{t}_{o}=\left(t_{1}, t_{3}, \ldots\right)$, series (1) is a BKP $\tau$-function, where the set $\mathbf{t}_{o}$ are higher BKP times. 
The second set of parameters $\mathbf{t}_{o}^{*}=\left(t_{1}^{*}, t_{3}^{*}, \ldots\right)$ give the evolution in a second BKP hierarchy.

REMARK 1. Consider a set $S$, which consists of distinct non-negative integers and includes zero. By $\mathrm{DP}_{S}$ we denote the subset of all strict partitions whose parts belong to the set $S$. By the limiting procedure: $e^{\xi k} \rightarrow 0$ iff $k$ does not belong to the set $S$, we can restrict the sum in (1) to the set $\mathrm{DP}_{S}$. If $S$ is a finite set, then (1) is a polynomial in $\mathbf{t}_{o}$ which describes a rational solution of the BKP hierarchy.

The typical choice of the BKP higher times is the following:

$$
m t_{m}=\sum_{k}^{N}\left(x_{k}^{m}-\left(-x_{k}\right)^{m}\right), \quad m t_{m}^{*}=\sum_{k}^{N^{*}}\left(y_{k}^{m}-\left(-y_{k}\right)^{m}\right) .
$$

In this case the sum in (1) ranges over all partitions whose length do not exceed $k=\min \left(N, N^{*}\right)$.

We note that a special case of this series (1), where times were chosen as in (2), and $e^{\xi_{m}}$ was chosen as a step function, was considered in [16] in a combinatorial context, not related to integrable systems.

In the present paper, we will specialize the variables $\mathbf{t}_{o}^{*}$ as $\mathbf{t}_{\infty}=(1,0,0, \ldots)$, and study the $\tau$-function (1) as a function of the variables $\xi_{m}$. We find that series (1) is a multi-soliton $\tau$-function of a different integrable hierarchy, which we call the dual BKP hierarchy. The variables $\xi_{m}$ of (1) turn out to be linear combinations of the time variables $\tilde{\mathbf{t}}_{o}=\left(\tilde{t}_{1}, \tilde{t}_{3}, \ldots\right)$ of the dual BKP hierarchy. We observe that the variables $\xi_{m}$ (proportional to the times of the dual BKP hierarchy) are integrals of motion of the original BKP hierarchy and, simultaneously, the times $\mathbf{t}_{o}$ of the original BKP hierarchy are integrals of motion of the dual BKP hierarchy. That is why we call these hierarchies dual to one another.

The situation we will describe is closely related to corresponding results for the hypergeometric $\tau$-functions of the KP hierarchy. These $\tau$-functions are described as hypergeometric because they generalize some known hypergeometric functions of many variables, see $[18,19]$. We note that the KP hypergeometric $\tau$-functions yields a perturbative asymptotic expansion for a set of known matrix integrals [10, 27]. They were also used to construct new solvable matrix integrals [28, 29]. Other examples of hypergeometric $\tau$-functions arise in supersymmetric gauge theories [20], [21], in the problem of counting of Hurwitz numbers [22], in counting Gromov-Witten invariants of $P^{1}$ [23] and in the computation of intersection numbers on Hilbert schemes [24]. In references [30,31], $\tau$-functions, which were considered in the context of $c=1$ strings, are also of hypergeometric type. The series for two dimensional QCD, considered in $[\mathbf{2 5}, \mathbf{2 6}]$, may be related to the KP hypergeometric $\tau$-functions also. We anticipate that applications of similar series in $Q$-functions are found also.

The series (1) can be studied in the context of random strict partitions. Series (1) generalizes the sums over random partitions which are considered in [16].

With regard to notation used in this paper, we will use infinite sequences of higher times

$$
\begin{aligned}
\mathbf{t} & =\left(t_{1}, t_{2}, t_{3}, \ldots\right), & \mathbf{t}^{*} & =\left(t_{1}^{*}, t_{2}^{*}, t_{3}^{*}, \ldots\right), \\
\mathbf{t}_{o} & =\left(t_{1}, t_{3}, t_{5}, \ldots\right), & \mathbf{t}_{o}^{*} & =\left(t_{1}^{*}, t_{3}^{*}, t_{5}^{*} \ldots\right),
\end{aligned}
$$


and, when they appear as higher times in dual equations will be marked with a tilde. Special cases of these, $\mathbf{t}_{\infty}$ and $\mathbf{t}_{o}(q)$, will be defined in (80) and (81) below.

2. KP and BKP $\boldsymbol{\tau}$-functions. In this section we will summarise the essential facts about $\tau$-functions for the KP and BKP hierarchies as given in [1]. The definitions of terms related to symmetric functions may be found in [6].

2.1. Schur functions as KP $\boldsymbol{\tau}$-functions. Let $A$ be the complex Clifford algebra generated by the charged free fermions $\psi_{i}, \psi_{i}^{*}$, where $i \in \mathbb{Z}$ with anticommutation relations

$$
\left[\psi_{i}, \psi_{j}\right]_{+}=\left[\psi_{i}^{*}, \psi_{j}^{*}\right]_{+}=0, \quad\left[\psi_{i}, \psi_{j}^{*}\right]_{+}=\delta_{i, j}
$$

Consider also the generators

$$
\psi(p)=\sum_{k \in \mathbb{Z}} \psi_{k} p^{k}, \quad \psi^{*}(q)=\sum_{k \in \mathbb{Z}} \psi_{k}^{*} q^{-k-1} .
$$

The vacuum expectation value is a linear functional \langle\rangle$: A \rightarrow \mathbb{C}$. For linear and quadratic elements in $A$ it is defined by $\left\langle\psi_{i}\right\rangle=\left\langle\psi_{i}^{*}\right\rangle=\left\langle\psi_{i} \psi_{j}\right\rangle=\left\langle\psi_{i}^{*} \psi_{j}^{*}\right\rangle=0$ and

$$
\left\langle\psi_{i} \psi_{j}^{*}\right\rangle=\left\{\begin{array}{ll}
\delta_{i, j} & i<0 \\
0 & i \geq 0
\end{array}, \quad\left\langle\psi_{i}^{*} \psi_{j}\right\rangle=\left\{\begin{array}{ll}
\delta_{i, j} & i \geq 0 \\
0 & i<0
\end{array} .\right.\right.
$$

For an arbitrary product of linear terms in $A$, Wick's Theorem gives

$$
\begin{aligned}
\left\langle 0\left|w_{1} \cdots w_{2 n+1}\right| 0\right\rangle & =0 \\
\left\langle 0\left|w_{1} \cdots w_{2 n}\right| 0\right\rangle & =\sum_{\sigma} \operatorname{sgn}(\sigma)\left\langle 0\left|w_{\sigma(1)} w_{\sigma(2)}\right| 0\right\rangle \cdots\left\langle 0\left|w_{\sigma(2 n-1)} w_{\sigma(2 n)}\right| 0\right\rangle,
\end{aligned}
$$

where $w_{k}$ are linear terms in $A$, and $\sigma$ runs over permutations such that $\sigma(1)<$ $\sigma(2), \ldots, \sigma(2 n-1)<\sigma(2 n)$, and $\sigma(1)<\sigma(3)<\cdots<\sigma(2 n-1)$.

The connection between the anticommutation relations and the vacuum expectation value is that $\left[w_{1}, w_{2}\right]_{+}=\left\langle w_{1} w_{2}\right\rangle+\left\langle w_{2} w_{1}\right\rangle$.

For free fermion generators with $|p| \neq|q|$,

$$
\left\langle\psi(p) \psi^{*}(q)\right\rangle=\frac{1}{p-q},
$$

and for higher degree products, Wick's Theorem gives

$$
\begin{aligned}
\left\langle\psi\left(p_{1}\right) \psi^{*}\left(q_{1}\right) \cdots \psi\left(p_{n}\right) \psi^{*}\left(q_{n}\right)\right\rangle & =\operatorname{det}\left(\left\langle\psi\left(p_{i}\right) \psi^{*}\left(q_{j}\right)\right\rangle\right) \\
& =\prod_{i} \frac{1}{p_{i}-q_{i}} \prod_{i<j} \frac{\left(p_{i}-p_{j}\right)\left(q_{i}-q_{j}\right)}{\left(p_{i}-q_{j}\right)\left(q_{i}-p_{j}\right)} .
\end{aligned}
$$

Time evolution enters $A$ via the hamiltonian

$$
H(\mathbf{t})=\sum_{n=1}^{\infty} H_{n} t_{n}
$$


where

$$
H_{n}=\sum_{k \in \mathbb{Z}} \psi_{k} \psi_{k+n}^{*}
$$

For any $a \in A$, we define

$$
a(\mathbf{t})=e^{H(\mathbf{t})} a e^{-H(\mathbf{t})}=\exp (\operatorname{ad} H(\mathbf{t})) a,
$$

and it may be shown that

$$
\psi(p)(\mathbf{t})=\exp (\xi(p, \mathbf{t})) \psi(p), \quad \psi^{*}(q)(\mathbf{t})=\exp (-\xi(q, \mathbf{t})) \psi^{*}(q),
$$

where $\xi(p, \mathbf{t})=\sum_{k=1}^{\infty} p^{k} t_{k}$.

Consider $g \in A$, which solves the following bilinear equation

$$
\left[g \otimes g, \sum_{n=-\infty}^{\infty} \psi_{n} \otimes \psi_{n}^{*}\right]=0
$$

where the notation [, ] stands for the commutator, and $\otimes$ is the tensor product. Then, one has a $\tau$-function

$$
\tau(\mathbf{t})=\langle g(\mathbf{t})\rangle
$$

The simplest type of $\tau$-functions correspond to multi-soliton solution of the KP hierarchy. Taking $g=\exp \left(\sum_{i=1}^{n} a_{i} \psi\left(p_{i}\right) \psi^{*}\left(q_{i}\right)\right)$ gives the $n$-soliton $\tau$-function

$$
\tau(\mathbf{t})=\operatorname{det}\left(\delta_{i, j}+\frac{a_{i}}{p_{i}-q_{j}} e^{\xi\left(p_{i}, \mathbf{t}\right)-\xi\left(q_{j}, \mathbf{t}\right)}\right),
$$

where

$$
\xi(p, \mathbf{t})=\sum_{m=1}^{\infty} p^{m} t_{m}
$$

Later, we will also need the soliton solution of the two-dimensional Toda lattice equation (TL) [32], which is described by almost the same formula

$$
\tau\left(n, \mathbf{t}, \mathbf{t}^{*}\right)=A\left(\mathbf{t}, \mathbf{t}^{*}\right) \operatorname{det}\left(\delta_{i, j}+\frac{a_{i}}{p_{i}-q_{j}} e^{\xi\left(p_{i}, n, \mathbf{t}, \mathbf{t}^{*}\right)-\xi\left(q_{j}, n, \mathbf{t}, \mathbf{t}^{*}\right)}\right),
$$

where

$$
\xi\left(p, n, \mathbf{t}, \mathbf{t}^{*}\right)=\sum_{m=1}^{\infty}\left(p^{m} t_{m}-p^{-m} t_{m}^{*}\right)+n \log p
$$

and

$$
A\left(\mathbf{t}, \mathbf{t}^{*}\right)=e^{\sum_{m=1}^{\infty} m t_{m} t_{m}^{*}}
$$

which is usually omitted from the definition of the TL $\tau$-function [32] since the transformation to nonlinear variables removes it from the TL solution. We will also neglect this term for the same reason. It is well-known [32], that any TL $\tau$-function 
is a $\tau$-function of the pair of KP hierarchies with higher times respectively $\mathbf{t}$ and $\mathbf{t}^{*}$. There exists a reduction to the one-dimensional TL, which yields also a reduction to the nonlinear Schrödinger equation. This reduction is described by the demand that the $\tau$-function of the two-dimensional TL (up to the irrelevant factor (21), depends only on $\mathbf{t}+\mathbf{t}^{*}$. It is provided by the condition $q_{i}=p_{i}^{-1}$ in (19) and we will use it in what follows. We shall also use the reduction to $\mathrm{KdV}$, namely the choice $q_{i}=-p_{i}$ in (17). $\mathrm{The} \mathrm{KdV} \tau$-function depends only on the odd index KP higher times, that is, on the sequence $\mathbf{t}_{o}$.

Polynomial $\tau$-functions are obtained by considering expansions in the parameters $p_{i}$ and $q_{j}$. First, elementary Schur polynomials $s_{i}(\mathbf{t})$ are defined by

$$
\exp (\xi(p, \mathbf{t}))=\sum_{k \geq 0} s_{i}(\mathbf{t}) p^{k}
$$

Since

$$
1=\exp (\xi(p, \mathbf{t})) \exp (-\xi(p, \mathbf{t}))=\sum_{i \geq 0} \sum_{j=0}^{i} s_{i-j}(\mathbf{t}) s_{j}(-\mathbf{t}) p^{i}
$$

we have the orthogonality condition

$$
\sum_{j=0}^{i} s_{i-j}(\mathbf{t}) s_{j}(-\mathbf{t})=\delta_{i, 0}
$$

For all non-negative integers we can define

$$
s_{(a \mid b)}(\mathbf{t})=(-1)^{b} \sum_{k=0}^{b} s_{a+1+k}(\mathbf{t}) s_{b-k}(-\mathbf{t})=(-1)^{b+1} \sum_{k=0}^{a} s_{k}(\mathbf{t}) s_{a+b+1-k}(-\mathbf{t}) .
$$

This is the Schur function for the partition $\left(a+1, b^{j}\right)$, which is written using Frobenius notation as $(a \mid b)$. This result is easily proved using the Jacobi-Trudi identity. For any partition function written in Frobenius notation,

$$
s_{\left(a_{1} a_{2} \ldots a_{n} \mid b_{1} b_{2} \ldots b_{n}\right)}(\mathbf{t})=\operatorname{det}\left(s_{\left(a_{i} \mid b_{j}\right)}\right) .
$$

Using this notation, (14) gives

$$
\psi_{i}(\mathbf{t})=\sum_{k \geq 0} s_{k}(\mathbf{t}) \psi_{i-k}, \quad \psi_{i}^{*}(\mathbf{t})=\sum_{k \geq 0} s_{k}(-\mathbf{t}) \psi_{i+k}^{*} .
$$

Consequently,

$$
\left\langle\psi_{i}(\mathbf{t}) \psi_{j}^{*}(\mathbf{t})\right\rangle=\sum_{k, \ell \geq 0} s_{k}(\mathbf{t}) s_{\ell}(-\mathbf{t})\left\langle\psi_{i-k} \psi_{j+\ell}^{*}\right\rangle=\sum_{k=i+1}^{i-j} s_{k}(\mathbf{t}) s_{i-j-k}(-\mathbf{t}) .
$$

Hence we see that

$$
s_{(a \mid b)}(\mathbf{t})=(-1)^{b+1}\left\langle\psi_{a}(\mathbf{t}) \psi_{-b-1}^{*}(\mathbf{t})\right\rangle,
$$


that is that $s_{(a \mid b)}$ is the $\mathrm{KP} \tau$-function for $g=(-1)^{b+1} \psi_{a} \psi_{-b-1}^{*}$. More generally, this shows that an arbitrary Schur function $s_{\left(a_{1} \cdots a_{n} \mid b_{1} \cdots b_{n}\right)}$, is a KP $\tau$-function for

$$
g=(-1)^{b_{1}+\cdots+b_{n}+n} \psi_{a_{1}} \psi_{-b_{1}-1}^{*} \cdots \psi_{a_{n}} \psi_{-b_{n}-1}^{*} .
$$

2.2. $Q$-functions as BKP $\tau$-functions. The subalgebra of $A$ invariant under the symmetry

$$
\psi_{i} \leftrightarrow(-1)^{i} \psi_{-i}^{*}
$$

is used in a similar way to determine BKP $\tau$-functions. There are two bases of neutral free fermions

$$
\phi_{i}=\frac{1}{\sqrt{2}}\left(\psi_{i}+(-1)^{i} \psi_{-i}^{*}\right), \quad \hat{\phi}_{i}=\frac{i}{\sqrt{2}}\left(\psi_{i}-(-1)^{i} \psi_{-i}^{*}\right),
$$

where $i \in \mathbb{Z}$, each of which generates this subalgebra.

Using the results for charged free fermions, the anticommutation relations are

$$
\left[\phi_{i}, \phi_{j}\right]_{+}=\left[\hat{\phi}_{i}, \hat{\phi}_{j}\right]_{+}=(-1)^{i} \delta_{i,-j}, \quad\left[\phi_{i}, \hat{\phi}_{j}\right]_{+}=0,
$$

and, in particular, $\phi_{0}^{2}=\hat{\phi}_{0}^{2}=\frac{1}{2}$. Similarly, the vacuum expectation values of quadratic elements are given by

$$
\left\langle\phi_{i} \phi_{j}\right\rangle=\left\langle\hat{\phi}_{i} \hat{\phi}_{j}\right\rangle= \begin{cases}(-1)^{i} \delta_{i,-j} & i<0 \\ \frac{1}{2} \delta_{j, 0} & i=0 \\ 0 & i>0\end{cases}
$$

and Wick's Theorem is used for arbitrary degree products.

The neutral free fermion generator is defined by $\phi(p)=\sum_{n \in \mathbb{Z}} p^{n} \phi_{n}$. We have (for $\left.|p| \neq\left|p^{\prime}\right|\right)$

$$
\left\langle\phi(p) \phi\left(p^{\prime}\right)\right\rangle=\frac{1}{2} \frac{p-p^{\prime}}{p+p^{\prime}},
$$

and $\left\langle\phi\left(p^{\prime}\right) \phi(p)\right\rangle=-\left\langle\phi(p) \phi\left(p^{\prime}\right)\right\rangle$. By Wick's Theorem we get

$\left\langle\phi\left(p_{1}\right) \phi\left(p_{2}\right) \cdots \phi\left(p_{N}\right)\right\rangle=\left\{\begin{array}{ll}\operatorname{Pf}\left(\left\langle\phi\left(p_{i}\right) \phi\left(p_{j}\right)\right\rangle\right) \\ 0\end{array}=\left\{\begin{array}{ll}2^{-N / 2} \prod_{i<j}^{\frac{p_{i}-p_{j}}{p_{i}+p_{j}}} & N \text { even } \\ 0 & \text { otherwise }\end{array}\right.\right.$.

The connection between the charged and neutral free fermions can be expressed in terms of the generators as

$$
-q \psi(p) \psi^{*}(-q)+p \psi(q) \psi^{*}(-p)=\phi(p) \phi(q)+\hat{\phi}(p) \hat{\phi}(q) .
$$

In the BKP reduction, even times are set equal to zero and we define $\mathbf{t}_{o}=$ $\left(t_{1}, 0, t_{3}, 0, t_{5}, \ldots\right)$, and the hamiltonian

$$
H^{B}\left(\mathbf{t}_{o}\right)=\sum_{i \geq 1, \text { odd }} H_{n}^{B} t_{n}
$$


where

$$
H_{n}^{B}=\frac{1}{2} \sum_{i \in \mathbb{Z}}(-1)^{i+1} \phi_{i} \phi_{-i-n} .
$$

For the fermion generating function one has

$$
\phi(p)\left(\mathbf{t}_{o}\right)=e^{H^{B}\left(\mathbf{t}_{o}\right)} \phi(p) e^{-H^{B}\left(\mathbf{t}_{o}\right)}=e^{\hat{H}^{B}\left(\mathbf{t}_{o}\right)} \phi(p) e^{-\hat{H}^{B}\left(\mathbf{t}_{o}\right)}=e^{\xi\left(p, \mathbf{t}_{o}\right)} \phi(p) .
$$

Note also that

$$
H\left(\mathbf{t}_{o}\right)=H^{B}\left(\mathbf{t}_{o}\right)+\hat{H}^{B}\left(\mathbf{t}_{o}\right), \quad\left[H^{B}\left(\mathbf{t}_{o}\right), \hat{H}^{B}\left(\mathbf{t}_{o}\right)\right]=0 .
$$

Similar to the KP case, BKP $\tau$-functions are defined by

$$
\tau_{B}\left(\mathbf{t}_{o}\right)=\left\langle h\left(\mathbf{t}_{o}\right)\right\rangle,
$$

where $h$ is the Clifford algebra of the neutral free fermions $\phi_{i}$. The $n$-soliton $\tau$-function is obtained by the choice $g=\exp \left(\sum_{i=1}^{n} a_{i} \phi\left(p_{i}\right) \phi\left(q_{i}\right)\right)$.

The Schur $q$ polynomials are defined by

$$
\exp \left(2 \xi\left(p, \mathbf{t}_{o}\right)\right)=\sum_{k \geq 0} q_{k}\left(\mathbf{t}_{o}\right) p^{k} .
$$

Thus

$$
\phi_{i}\left(\mathbf{t}_{o}\right)=\sum_{k \geq 0} q_{k}\left(\frac{1}{2} \mathbf{t}_{o}\right) \phi_{i-k}
$$

We have

$$
\left\langle\phi_{i}\left(\mathbf{t}_{o}\right) \phi_{j}\left(\mathbf{t}_{o}\right)\right\rangle=\frac{1}{2} q_{i}\left(\frac{1}{2} \mathbf{t}_{o}\right) q_{j}\left(\frac{1}{2} \mathbf{t}_{o}\right)+\sum_{k=1}^{j}(-1)^{k} q_{k+i}\left(\frac{1}{2} \mathbf{t}_{o}\right) q_{j-k}\left(\frac{1}{2} \mathbf{t}_{o}\right) .
$$

Since

$$
1=\exp \left(2 \xi\left(p, \mathbf{t}_{o}\right)\right) \exp \left(-2 \xi\left(p, \mathbf{t}_{o}\right)\right)=\sum_{i, j} q_{i}\left(\mathbf{t}_{o}\right) q_{j-i}\left(-\mathbf{t}_{o}\right)=\sum_{i, j}(-1)^{i-j} q_{i}\left(\mathbf{t}_{o}\right) q_{j-i}\left(\mathbf{t}_{o}\right) p^{j},
$$

for all $n>0$ we have

$$
\sum_{i=0}^{n}(-1)^{i} q_{i}\left(\mathbf{t}_{o}\right) q_{n-i}\left(\mathbf{t}_{o}\right)=0 .
$$

This is trivial if $n$ is odd and if $n=2 m$ is even then it gives

$$
q_{m}\left(\mathbf{t}_{0}\right)^{2}+2 \sum_{k=1}^{m}(-1)^{k} q_{m+k}\left(\mathbf{t}_{o}\right) q_{m-k}\left(\mathbf{t}_{o}\right)=0 .
$$

We can also define

$$
q_{a, b}\left(\mathbf{t}_{o}\right)=q_{a}\left(\mathbf{t}_{o}\right) q_{b}\left(\mathbf{t}_{o}\right)+2 \sum_{k=1}^{b}(-1)^{k} q_{a+k}\left(\mathbf{t}_{o}\right) q_{b-k}\left(\mathbf{t}_{o}\right)
$$


If follows from the orthogonality condition (46) that

$$
q_{a, b}\left(\mathbf{t}_{o}\right)=-q_{b, a}\left(\mathbf{t}_{o}\right),
$$

and in particular, $q_{a, a}\left(\mathbf{t}_{o}\right)=0$. Comparing (44) and (48), it is clear that

$$
q_{a, b}\left(\frac{1}{2} \mathbf{t}_{o}\right)=2\left\langle\phi_{a}\left(\mathbf{t}_{o}\right) \phi_{b}\left(\mathbf{t}_{o}\right)\right\rangle \text {. }
$$

Now consider $\lambda=\left(\lambda_{1}, \lambda_{2}, \cdots, \lambda_{2 n}\right)$ where $\lambda_{1}>\lambda_{2}>\cdots \lambda_{2 n-1}>\lambda_{2 n} \geq 0$. Note that this is a partition with an extra trivial part 0 included if necessary to ensure that the number of parts is even. The set of such strict, or distinct part, partitions is denoted DP. For $\lambda \in \mathrm{DP}$ we define

$$
Q_{\lambda}\left(\frac{1}{2} \mathbf{t}_{o}\right)=\operatorname{Pf}\left(q_{\lambda_{i}, \lambda_{j}}\left(\frac{1}{2} \mathbf{t}_{o}\right)\right) .
$$

This is the Schur $Q$-function. By Wick's theorem,

$$
Q_{\lambda}\left(\frac{1}{2} \mathbf{t}_{o}\right)=\operatorname{Pf}\left(2\left\langle\phi_{\lambda_{i}}\left(\mathbf{t}_{o}\right) \phi_{\lambda_{j}}\left(\mathbf{t}_{o}\right)\right\rangle\right)=2^{n}\left\langle\phi_{\lambda_{1}}\left(\mathbf{t}_{o}\right) \phi_{\lambda_{2}}\left(\mathbf{t}_{o}\right) \cdots \phi_{\lambda_{2 n}}\left(\mathbf{t}_{o}\right)\right\rangle .
$$

2.3. Hypergeometric $\tau$-functions. These $\tau$-functions were introduced by one of the authors in the KP case [8] and the BKP case [9].

In the KP case, let $r$ be a function of one variable and for any partition $\lambda$, let $r_{\lambda}(x)=\prod_{(i, j) \in \lambda} r(x-i+j)$, the product being over all vertices in the Young diagram. Then

$$
\tau\left(n, \mathbf{t}, \mathbf{t}^{*}\right)=\sum_{\lambda} r_{\lambda}(n) s_{\lambda}(\mathbf{t}) s_{\lambda}\left(\mathbf{t}^{*}\right)
$$

where $\mathbf{t}=\left(t_{1}, t_{2}, t_{3}, \ldots\right), \mathbf{t}^{*}=\left(t_{1}^{*}, t_{2}^{*}, t_{3}^{*}, \ldots\right)$, is the KP hypergeometric $\tau$-function.

In the BKP case, $r_{\lambda}$ has a different definition: if $\lambda=\left(\lambda_{1}, \ldots, \lambda_{k}\right)$ then $r_{\lambda}=$ $\prod_{i=1}^{k} r(1) r(2) \cdots r\left(\lambda_{i}\right)$. If we introduce new variables $r(k)=e^{\xi_{k}-\xi_{k-1}}, \quad \xi_{-1}=0$ then $r_{\lambda}=\prod_{i=1}^{\ell(\lambda)} e^{\xi_{\lambda i}}$

With these definitions,

$$
\tau\left(\mathbf{t}_{o}, \xi, \mathbf{t}_{o}^{*}\right)=\sum_{\lambda \in \mathrm{DP}} 2^{-\ell(\lambda)} r_{\lambda} Q_{\lambda}\left(\frac{1}{2} \mathbf{t}_{o}\right) Q_{\lambda}\left(\frac{1}{2} \mathbf{t}_{o}^{*}\right)=\sum_{\lambda \in \mathrm{DP}} 2^{-\ell(\lambda)} \prod_{i=1}^{\ell(\lambda)} e^{\xi_{\lambda_{i}}} Q_{\lambda}\left(\frac{1}{2} \mathbf{t}_{o}\right) Q_{\lambda}\left(\frac{1}{2} \mathbf{t}_{o}^{*}\right)
$$

is the BKP hypergeometric $\tau$-function. By Remark 1 one can restrict sum (53) to the sum which ranges over $\mathrm{DP}_{S}$.

It can be show that this is a $\tau$-function since

$$
\tau\left(\mathbf{t}_{o}, \xi, \mathbf{t}_{o}^{*}\right)=\left\langle 0\left|e^{\sum_{i \geq 1, \text { odd }} H_{n}^{B} t_{n}} e^{\sum_{n=-\infty}^{\infty}(-)^{n} \xi_{n}: \phi_{n} \phi_{-n}:} e^{\sum_{i \geq 1, \text { odd }} H_{-n}^{B} t_{n}^{*}}\right| 0\right\rangle
$$

\section{Main results.}

3.1. KP infinite soliton solution. Let

$$
g^{\mathrm{sol}}=\exp \left(\sum_{i, j \geq 0} a_{i, j} \psi\left(p_{i}\right) \psi^{*}\left(q_{j}\right)\right)
$$


Then

$$
\begin{aligned}
\tau^{\mathrm{sol}}:= & \left\langle g^{\mathrm{sol}}(\mathbf{t})\right\rangle \\
= & 1+\sum_{i, j} a_{i, j}\left\langle\psi\left(p_{i}\right) \psi^{*}\left(q_{j}\right)(\mathbf{t})\right\rangle+\sum_{i, j, k, l}\left(a_{i, j} a_{k, l}-a_{i, l} a_{j, k}\right)\left\langle\psi\left(p_{i}\right) \psi^{*}\left(q_{j}\right) \psi\left(p_{k}\right) \psi^{*}\left(q_{l}\right)(\mathbf{t})\right\rangle \\
& +\cdots=1+\sum_{i, j}\left(\begin{array}{l}
i \\
j
\end{array}\right) \frac{1}{p_{i}-q_{j}} e^{\xi\left(p_{i}, \mathbf{t}\right)-\xi\left(q_{j}, \mathbf{t}\right)} \\
& +\sum_{i, j, k, l}\left(\begin{array}{c}
i, j \\
k, l
\end{array}\right) \frac{1}{\left(p_{i}-q_{j}\right)\left(p_{k}-q_{l}\right)} \frac{\left(p_{i}-p_{k}\right)\left(q_{j}-q_{l}\right)}{\left(p_{i}-q_{l}\right)\left(q_{j}-p_{k}\right)} e^{\xi\left(p_{i}, \mathbf{t}\right)-\xi\left(q_{j}, \mathbf{t}\right)+\xi\left(p_{k}, \mathbf{t}\right)-\xi\left(q_{l}, \mathbf{t}\right)}+\cdots,
\end{aligned}
$$

where

$$
\left(\begin{array}{c}
i, j \\
k, l
\end{array}\right)
$$

denotes the $2 \times 2$ minor of the infinite matrix $\left(a_{i j}\right)$ containing the $i$ th and $j$ th rows and the $k$ th and $l$ th columns.

If $a_{i, j}=s_{(i \mid j)}\left(\mathbf{t}^{*}\right)$ then the coefficients can be written as

$$
s_{\lambda}\left(\mathbf{t}^{*}\right)
$$

for all partitions $\lambda$.

3.2. KdV soliton solution. The KdV reduction of the KP soliton solution (17),

$$
g_{\mathrm{KdV}}^{\mathrm{sol}}=\exp \left(\sum_{i \geq 0} a_{i} p_{i} \psi\left(p_{i}\right) \psi^{*}\left(-p_{i}\right)\right)
$$

gives rise to the following soliton $\tau$-function, which depends only on the higher KP times with odd numbers $\mathbf{t}_{o}=\left(t_{1}, t_{3}, t_{5}, \ldots\right)$,

$$
\begin{aligned}
\tau_{\mathrm{KdV}}^{\mathrm{sol}}:= & \left\langle g_{\mathrm{KdV}}^{\mathrm{sol}}\left(\mathbf{t}_{o}\right)\right\rangle=\operatorname{det}\left(\delta_{i, j}+\frac{a_{i} p_{i}}{p_{i}+p_{j}} e^{\xi\left(p_{i}, \mathbf{t}_{o}\right)-\xi\left(-p_{i}, \mathbf{t}_{o}\right)}\right) \\
= & 1+\sum_{i} \frac{1}{2} e^{\eta_{i}}+\sum_{i>j} \frac{1}{2^{2}} \frac{\left(p_{i}-p_{j}\right)^{2}}{\left(p_{i}+p_{j}\right)^{2}} e^{\eta_{i}+\eta_{j}} \\
& +\sum_{i>j>k} \frac{1}{2^{3}} \frac{\left(p_{i}-p_{j}\right)^{2}\left(p_{i}-p_{k}\right)^{2}\left(p_{j}-p_{k}\right)^{2}}{\left(p_{i}+p_{j}\right)^{2}\left(p_{i}+p_{k}\right)^{2}\left(p_{j}+p_{k}\right)^{2}} e^{\eta_{i}+\eta_{j}+\eta_{k}}+\cdots,
\end{aligned}
$$

where

$$
\eta_{i}=2 \sum_{m=1}^{\infty} t_{2 m-1} p_{i}^{2 m-1}+\log a_{i}, \quad i=0,1,2, \ldots
$$


REMARK 2. We note that the fractional linear transformation of the (complex) plane of spectral parameters $p_{i} \in \mathbb{C}$

$$
p_{i} \rightarrow \frac{a p_{i}+b}{c p_{i}+d}, \quad a=d=0, \quad b, c \neq 0 \quad \text { or } \quad a, d \neq 0, \quad b=c=0, \quad i=1,2, \ldots
$$

leaves invariant the factors

$$
\frac{\left(p_{i}-p_{j}\right)^{2}}{\left(p_{i}+p_{j}\right)^{2}}, \quad i, j=1,2, \ldots
$$

3.3. NLS and one-dimensional Toda lattice soliton $\tau$-function. Let us consider the reduction of the TL soliton $\tau$-function (19) to the one-dimensional Toda lattice (1DTL) reduction, which is $q_{i}=p_{i}^{-1}$, see [32]. If, in addition, $\left|p_{i}\right|=1$, then, it is also a reduction to the nonlinear Schrödinger equation (NLS). For the multi-soliton tau function we have

$$
\begin{gathered}
g_{1 \mathrm{DTL}}^{\mathrm{sol}}=\exp \left(\sum_{i \geq 0} \frac{a_{i}}{2}\left(p_{i}-p_{i}^{-1}\right) \psi\left(p_{i}\right) \psi^{*}\left(p_{i}^{-1}\right)\right) \\
\tau_{1 \mathrm{DTL}}^{\mathrm{sol}}\left(n, \mathbf{t}, \mathbf{t}^{*}\right)=\tau_{1 \mathrm{DTL}}^{\mathrm{sol}}\left(n, \mathbf{t}+\mathbf{t}^{*}\right):= \\
=\left\langle g_{1 \mathrm{DTL}}^{\mathrm{sol}}\left(n, \mathbf{t}, \mathbf{t}^{*}\right)\right\rangle=\operatorname{det}\left(\delta_{i, j}+\frac{a_{i}\left(p_{i}-p_{i}^{-1}\right)}{2\left(p_{i}-p_{j}^{-1}\right)} e^{\xi\left(p_{i}, n, \mathbf{t}, \mathbf{t}^{*}\right)-\xi\left(p_{j}^{-1}, n, \mathbf{t}, \mathbf{t}^{*}\right)}\right) \\
=1+\sum_{i} \frac{1}{2} e^{\eta_{i}}+\sum_{i>j} \frac{1}{2^{2}} \frac{\left(p_{i}-p_{j}\right)^{2}}{\left(p_{i} p_{j}-1\right)^{2}} e^{\eta_{i}+\eta_{j}} \\
+\sum_{i>j>k} \frac{1}{2^{3}} \frac{\left(p_{i}-p_{j}\right)^{2}\left(p_{i}-p_{k}\right)^{2}\left(p_{j}-p_{k}\right)^{2}}{\left(p_{i} p_{j}-1\right)^{2}\left(p_{i} p_{k}-1\right)^{2}\left(p_{j} p_{k}-1\right)^{2}} e^{\eta_{i}+\eta_{j}+\eta_{k}}+\cdots,
\end{gathered}
$$

where $\mathbf{t}=\left(t_{1}, t_{2}, t_{3}, \ldots\right), \mathbf{t}^{*}=\left(t_{1}^{*}, t_{2}^{*}, t_{3}^{*}, \ldots\right)$, and

$$
\eta_{i}=\sum_{m=1}^{\infty}\left(p_{i}^{m}-p_{i}^{-m}\right)\left(t_{m}+t_{m}^{*}\right)+2 n \log p_{i}+\log a_{i}, \quad i=0,1,2, \ldots
$$

For the nonlinear Schrödinger equation the $n$-dependence of the $\tau$-function is irrelevant.

REMARK 3. Here, the fractional linear transformations

$$
p_{i} \rightarrow \pm \frac{a p_{i}+b}{b p_{i}+a}, \quad i=0,1,2, \ldots
$$

where $a=0$ or $b=0$, but not both, leave invariant the factors

$$
\frac{\left(p_{i}-p_{j}\right)^{2}}{\left(p_{i} p_{j}-1\right)^{2}}, \quad i, j=0,1,2, \ldots
$$


3.4. BKP infinite soliton solution. Now writing $q_{i}=-p_{i}$ and choosing skewsymmetric matrix entries $a_{j i}=-a_{i j}$ in (55) gives

$$
g^{\mathrm{sol}}=\exp \left(\sum_{0 \leq i<j} a_{i, j}\left(-p_{j} \psi\left(p_{i}\right) \psi^{*}\left(-p_{j}\right)+p_{i} \psi\left(p_{j}\right) \psi^{*}\left(-p_{i}\right)\right)\right) .
$$

By (36) this may be rewritten as

$$
g^{\mathrm{sol}}=\exp \left(\sum_{0 \leq i<j} a_{i, j}\left(\phi\left(p_{i}\right) \phi\left(p_{j}\right)+\hat{\phi}\left(p_{i}\right) \hat{\phi}\left(p_{j}\right)\right)\right) .
$$

Since $\left[\phi_{i}, \hat{\phi}_{j}\right]_{+}=0,\left[\phi_{i} \phi_{k}, \hat{\phi}_{j} \hat{\phi}_{l}\right]=0$ and so we can factorize as $g=h \hat{h}$ where

$$
h=\exp \left(\sum_{i<j} a_{i, j} \phi\left(p_{i}\right) \phi\left(p_{j}\right)\right), \quad \hat{h}=\exp \left(\sum_{i<j} a_{i, j} \hat{\phi}\left(p_{i}\right) \hat{\phi}\left(p_{j}\right)\right) .
$$

Then, we have

$$
\begin{aligned}
\tau_{\mathrm{B}}^{\mathrm{sol}}\left(\mathbf{t}_{o}\right):= & \left\langle h\left(\mathbf{t}_{o}\right)\right\rangle=\left\langle\hat{h}\left(\mathbf{t}_{o}\right)\right\rangle \\
= & 1+\sum_{0 \leq i<j} a_{i, j}\left\langle\phi\left(p_{i}\right) \phi\left(p_{j}\right)\left(\mathbf{t}_{o}\right)\right\rangle \\
& +\sum_{0 \leq i<j<k<l}\left(a_{i, j} a_{k, l}-a_{i, k} a_{j, l}+a_{i, l} a_{j, k}\right)\left\langle\phi\left(p_{i}\right) \phi\left(p_{j}\right) \phi\left(p_{k}\right) \phi\left(p_{l}\right)\left(\mathbf{t}_{o}\right)\right\rangle+\cdots \\
= & 1+\sum_{0 \leq i<j}(i, j) \frac{1}{2} \frac{p_{i}-p_{j}}{p_{i}+p_{j}} e^{\xi\left(p_{i}, \mathbf{t}_{o}\right)+\xi\left(p_{j}, \mathbf{t}_{o}\right)} \\
& +\sum_{0 \leq i<j<k<l}(i, j, k, l) \frac{1}{2^{2}} \frac{\left(p_{i}-p_{j}\right)\left(p_{k}-p_{l}\right)}{\left(p_{i}+p_{j}\right)\left(p_{k}+p_{l}\right)} e^{\xi\left(p_{i}, \mathbf{t}_{o}\right)+\xi\left(p_{j}, \mathbf{t}_{o}\right)+\xi\left(p_{k}, \mathbf{t}_{o}\right)+\xi\left(p_{l}, \mathbf{t}_{o}\right)}+\cdots,
\end{aligned}
$$

where

$$
(i, j, k, l)
$$

denotes the pfaffian minor of the infinite skew-symmetric matrix $\left(a_{i j}\right)$ containing the $i$ th, $j$ th, $k$ th and $l$ th lines. Using (40) gives

$$
\tau^{\mathrm{sol}}\left(\mathbf{t}_{o}\right)=\tau_{B}^{\mathrm{sol}}\left(\mathbf{t}_{o}\right)^{2}
$$

If $a_{i, j}=q_{i, j}\left(\frac{1}{2} \mathbf{t}_{o}^{*}\right)$ then the coefficients can be written as

$$
Q_{\lambda}\left(\frac{1}{2} \mathbf{t}_{o}^{*}\right)
$$

for all partitions $\lambda$ into distinct parts.

REMARK 4. The factors

$$
(i, j) \frac{p_{i}-p_{j}}{p_{i}+p_{j}}, \quad(i, j, k, l) \frac{\left(p_{i}-p_{j}\right)\left(p_{k}-p_{l}\right)}{\left(p_{i}+p_{j}\right)\left(p_{k}+p_{l}\right)}, \ldots
$$


in (72), are invariant under the transformation

$$
\begin{gathered}
p_{i} \rightarrow \frac{a p_{i}+b}{c p_{i}+d}, \quad i=0,1,2, \ldots, \\
a_{i, j} \rightarrow \gamma a_{i, j}, \quad i, j=0,1,2, \ldots,
\end{gathered}
$$

where $a, b, c, d$ are complex numbers such that $a=d=0, b, c \neq 0$ or $a, d \neq 0, b=$ $c=0$

$$
\frac{a d+b c}{a d-b c}=\gamma(= \pm 1) .
$$

REMARK 5. Although the free fermion generator is not defined if its parameter is 0 , i.e. $\phi(0)$ does not make sense, the limit

$$
\lim _{p^{\prime} \rightarrow 0}\left\langle\phi(p) \phi\left(p^{\prime}\right)\right\rangle=\frac{1}{2}
$$

as given by (35), is well defined.

3.5. Useful Lemma. Let us introduce the following notation:

$$
\mathbf{t}_{\infty}=(1,0,0,0, \ldots)
$$

and

$$
\begin{gathered}
\mathbf{t}_{o}(q)=\left(t_{1}(q), t_{3}(q), t_{5}(q), \ldots\right), \quad t_{2 m-1}(q)=\frac{2}{(2 m-1)\left(1-q^{2 m-1}\right)} \\
t_{2 m}=0, m=1,2, \ldots
\end{gathered}
$$

REMARK 6 . Let us notice that $\mathbf{t}_{\infty}$ can be viewed as given by (2), where we take $x_{1}=x_{2}=\cdots=x_{N}=N^{-1}$ and $N \rightarrow \infty$. Similarly, $\mathbf{t}_{o}(q)$ is given by (2), where $x_{k}=$ $q^{k-1}, k=1,2, \ldots$ As for $\mathbf{t}_{\infty}$, if $f$ satisfies $f\left(c t_{1}, c^{3} t_{3}, c^{5} t_{5}, \ldots\right)=c^{d} f\left(t_{1}, t_{3}, t_{5}, \ldots\right)$ for some $d \in \mathbb{Z}$, we have $\hbar^{d} f\left(\mathbf{t}_{o}(q)\right) \rightarrow f\left(\mathbf{t}_{\infty}\right)$ as $\hbar:=\log q \rightarrow 0$. In that sense (80) may be considered as a limit of (81) as $q \rightarrow 1$.

We have the following result.

Lemma 1. Let $\lambda=\left(\lambda_{1}, \ldots, \lambda_{k}\right)$ be a strict partition. Then

$$
Q_{\lambda}\left(\frac{1}{2} \mathbf{t}_{\infty}\right)=\prod_{i=1}^{k} \frac{1}{\lambda_{i} !} \prod_{i<j}^{k} \frac{\lambda_{i}-\lambda_{j}}{\lambda_{i}+\lambda_{j}},
$$

and

$$
Q_{\lambda}\left(\frac{1}{2} \mathbf{t}_{o}(q)\right)=\prod_{i<j}^{k} \frac{q^{\lambda_{i}}-q^{\lambda_{j}}}{q^{\lambda_{j}+\lambda_{i}}-1} \prod_{i=1}^{k} \frac{(-q ; q)_{\lambda_{i}}}{(q ; q)_{\lambda_{i}}}
$$

where

$$
(p ; q)_{m}:=(1-p)(1-p q) \cdots\left(1-p q^{m-1}\right)
$$


3.6. Hypergeometric functions related to the projective Schur functions. We consider hypergeometric $\tau$-functions (1), where we specialize the variable $\mathbf{t}_{o}^{*}$ respectively by $(80)$ and (81):

$$
\begin{gathered}
\tau\left(\mathbf{t}_{o}, \xi, \mathbf{t}_{\infty}\right)=\sum_{\lambda \in \mathrm{DP}_{S}} \frac{Q_{\lambda}\left(\frac{1}{2} \mathbf{t}_{o}\right)}{2^{\ell(\lambda)} H_{\lambda}^{*}} \prod_{i=1}^{\ell(\lambda)} e^{\xi_{n i}}, \\
\tau\left(\mathbf{t}_{o}, \xi, \mathbf{t}_{o}(q)\right)=\sum_{\lambda \in \mathrm{DP}_{S}} \frac{Q_{\lambda}\left(\frac{1}{2} \mathbf{t}_{o}\right)}{2^{\ell(\lambda)} H_{\lambda}^{*}(q)} \prod_{i=1}^{\ell(\lambda)} e^{\xi_{n i}},
\end{gathered}
$$

where

$$
H_{\lambda}^{*}=Q_{\lambda}\left(\frac{1}{2} \mathbf{t}_{\infty}\right)^{-1}, \quad H_{\lambda}^{*}(q)=Q_{\lambda}\left(\frac{1}{2} \mathbf{t}_{o}(q)\right)^{-1}
$$

are respectively so-called product-of-shifted-hook-length [6], which generalize the notion of the factorial for strict partitions and its $q$-analog (shifted hook polynomial). We took into account Remark 1, to restrict sums over all strict partitions to the subset $\mathrm{DP}_{S}$.

In the case that $\mathrm{DP}_{S}$ is the set of all strict partitions, namely, DP, the series (85) and (86) may be considered as multi-variable generalization of hypergeometric function (respectively, basic hypergeometric function), which we obtain when $\ell(\lambda)=1$ and $\mathbf{t}_{o}$ is of form (2) where $N_{1}=1$.

The notation $Q\left(x^{(N)}\right)$ below will be used for $Q\left(\frac{\mathbf{t}_{\mathbf{o}}}{2}\right)$, where $\mathbf{t}_{o}$ is defined by (2). Let all parameters $b_{k}$ be not equal to negative integers. Let in (85) we choose

$$
e^{\xi_{n}}=\frac{\prod_{i=1}^{p} \Gamma\left(a_{i}+n\right) \Gamma\left(a_{i}\right)^{-1}}{\prod_{i=1}^{s} \Gamma\left(b_{i}+n\right) \Gamma\left(b_{i}\right)^{-1}}=\frac{\prod_{i=1}^{p}\left(a_{i}\right)_{n}}{\prod_{i=1}^{s}\left(b_{i}\right)_{n}}
$$

Then tau function (85) defines the following hypergeometric function

$$
{ }_{p} F_{s}\left(a_{1}, \ldots, a_{p} ; b_{1}, \ldots, b_{s} ; x^{(N)}\right):=\sum_{\substack{\lambda \in \mathrm{DP} \\ \ell(\lambda \leq N}}^{\infty} 2^{-\ell(\lambda)} \frac{\prod_{k=1}^{p}\left(a_{k}\right)_{\lambda}}{\prod_{k=1}^{s}\left(b_{k}\right)_{\lambda}} \frac{Q_{\lambda}\left(x^{(N)}\right)}{H_{\lambda}^{*}},
$$

which generalizes the hypergeometric function of one variable

$$
{ }_{p} F_{s}\left(a_{1}, \ldots, a_{p} ; b_{1}, \ldots, b_{s} ; x^{(1)}\right)=\sum_{n=0}^{\infty} \frac{\prod_{k=1}^{p}\left(a_{k}\right)_{n}}{\prod_{k=1}^{s}\left(b_{k}\right)_{n}} \frac{x^{n}}{n !}
$$

The function (89) was introduced in [9]. Here we introduce the $q$-deformed version of (89). If in (86) we choose

$$
e^{\xi_{n}}=\frac{\prod_{i=1}^{p}\left(q^{a_{i}} ; q\right)_{n}}{\prod_{i=1}^{s}\left(q^{b_{i}} ; q\right)_{n}}
$$

tau function (86) defines the hypergeometric function

$$
{ }_{p} \Phi_{s}\left(a_{1}, \ldots, a_{p} ; b_{1}, \ldots, b_{s} ; x^{(N)}\right):=\sum_{\substack{\lambda \in \mathrm{DP} \\ \ell(\lambda \leq N}}^{\infty} 2^{-\ell(\lambda)} \frac{\prod_{k=1}^{p}\left(q^{a_{k}} ; q\right)_{\lambda}}{\prod_{k=1}^{s}\left(q^{b_{k}} ; q\right)_{\lambda}} \frac{Q_{\lambda}\left(x^{(N)}\right)}{H_{\lambda}^{*}(q)}
$$


which generalizes the basic hypergeometric function of one variable

$$
{ }_{p} F_{s}\left(a_{1}, \ldots, a_{p} ; b_{1}, \ldots, b_{s} ; x^{(1)}\right)=\sum_{n=0}^{\infty} \frac{\prod_{k=1}^{p}\left(q^{a_{k}} ; q\right)_{n}}{\prod_{k=1}^{s}\left(q^{b_{k}} ; q\right)_{n}} \frac{x^{n}}{(q ; q)_{n}}
$$

Hypergeometric functions (89) and (92) may be also considered as multisoliton tau functions, see next subsection.

3.7. Soliton solutions and rational solutions. Let us recall, that in Remark 1 the set of the partitions $\mathrm{DP}_{S}$ was defined via a set of distinct non-negative integers $S$ which includes zero.

TheOREM 1. Let $\tau\left(\mathbf{t}_{o}, \xi, \mathbf{t}_{\infty}\right)$ be defined by (85), and $\tau^{\mathrm{sol}}\left(\mathfrak{\mathbf { t }}, \tilde{\mathbf{t}}^{*}\right)$ be defined by (72), where

$$
p_{m}=\frac{a m+b}{c m+d}, \quad m \in S
$$

(in particular, one can take integer momentum $p_{m}=m$ ), with $a, b, c, d$ such that $a=$ $d=0, b, c \neq 0$ or $a, d \neq 0, b=c=0$,

$$
\frac{a d+b c}{a d-b c}=\gamma,
$$

and

$$
a_{i, j}=\gamma q_{i, j}\left(\frac{1}{2} \mathbf{t}_{o}^{*}\right)
$$

Let for a given set of the numbers $p_{m}, m \in S$, the variables $\mathfrak{\mathbf { t }}, \mathbf{t}^{*}$ are related to the variables $\xi$ as

$$
\xi_{m}=\sum_{k=1}^{\infty}\left(p_{m}^{k} \tilde{t}_{k}-p_{m}^{-k} \tilde{t}_{k}^{*}\right)+\log m !
$$

Then we have

$$
\tau^{\mathrm{sol}}(\mathfrak{t})=\tau\left(\mathbf{t}_{o}, \xi, \mathbf{t}_{\infty}\right)
$$

Proof. Let us compare (85) and (72). First replace $\mathbf{t}_{o}$ with $\mathfrak{\mathbf { t }}_{o}$ in (72). Then a typical term on the right hand side is

$$
\left(n_{1}, n_{2}, \ldots, n_{k}\right) 2^{-k} \prod_{i<j} \frac{p_{n_{i}}-p_{n_{j}}}{p_{n_{i}}+p_{n_{j}}} \prod_{i=1}^{k} e^{\xi\left(p_{n_{i}}, \mathfrak{z}_{o}\right)},
$$

where $0 \leq n_{1}<n_{2}<\cdots<n_{k}$ and $k$ is even. We can set $\xi\left(p_{k}, \mathfrak{t}_{o}\right)=\xi_{k}$, choose the parameters $p_{k}=k$ and the pfaffian elements to be (for $i<j$ )

$$
(i, j)=\left\{\begin{array}{ll}
\frac{2 q_{j}\left(\frac{1}{2} \mathbf{t}_{o}\right)}{j !} & i=0 \\
\frac{q_{j, i}\left(\frac{1}{2} \mathbf{t}_{o}\right)}{i ! j !} & i>0
\end{array},\right.
$$


so that

$$
\left(n_{1}, n_{2}, \ldots, n_{k}\right)= \begin{cases}2 \prod_{i=1}^{k} \frac{1}{n_{k} !} Q_{\lambda}\left(\frac{1}{2} \mathbf{t}_{o}\right) & n_{1}=0 \\ \prod_{i=1}^{k} \frac{1}{n_{k} !} Q_{\lambda}\left(\frac{1}{2} \mathbf{t}_{o}\right) & n_{1}>0\end{cases}
$$

where $\lambda$ is the partition into distinct parts $\left(n_{k}, n_{k-1}, \ldots, n_{1}\right)$.

Thus the typical term may be written as

$$
2^{-\ell(\lambda)} \prod_{i=1}^{\ell(\lambda)} e^{\xi_{k}} Q_{\lambda}\left(\frac{1}{2} \mathbf{t}_{o}\right) Q_{\lambda}\left(\frac{1}{2} \mathbf{t}_{\infty}\right),
$$

for any partition into distinct parts. The partitions into an odd number of distinct parts come from those terms for which $n_{1}=0$. The extra factors of 2 in the pfaffian element in (100) and (101) are needed because in the case $n_{1}=0$, the length of the partition $\ell(\lambda)$ is $k-1$ not $k$.

This establishes the connection between (85) and (72).

The hypergeometric function (89) is an example of multi-soliton tau function of the dual BKP hierarchy, evaluated at special values of times $\mathfrak{t}_{o}$, see (88) and (97).

THEOREM 2. Tau functions $\tau\left(\mathbf{t}_{o}, \xi, \mathbf{t}_{\infty}\right)$ and $\tau_{\mathrm{KdV}}^{\mathrm{sol}}\left(\mathbf{t}_{o}\right)$ are defined respectively by (85) and by (60). Let us choose $p_{m}$ in (60) by

$$
p_{m}=\frac{a m+b}{c m+d}, \quad m \in S,
$$

where $a, b, c, d$ are such that $a=d=0, b, c \neq 0$ or $a, d \neq 0, b=c=0$,

(in particular, one can choose integer momentum $p_{m}=m, m \in S$ ). Let the numbers $\xi_{m}$ in $(85)$ be related to $\eta\left(\mathbf{t}_{o}, p_{m}\right)$ in (60) by

$$
\xi_{m}-\log m !=\eta_{m}:=2 \sum_{k=1}^{\infty} p_{m}^{2 k-1} \tilde{t}_{2 k-1}
$$

Then

$$
\tau_{\mathrm{KdV}}^{\mathrm{sol}}\left(\mathfrak{\mathbf { t }}_{o}\right)=\tau\left(\mathbf{t}_{\infty}, \xi, \mathbf{t}_{\infty}\right) .
$$

The hypergeometric function (89), where $x_{1}=x_{2}=\cdots=N^{-1}$ and $N \rightarrow \infty$, is an example of multi-soliton $\mathrm{KdV}$ tau function, evaluated at special values of times $\mathfrak{\mathbf { t }}_{o}$, see (88), (104) and Remark 6.

THEOREM 3. Tau functions $\tau\left(\mathbf{t}_{o}, \xi, \mathbf{t}_{o}(q)\right)$ and $\tau_{1 \mathrm{DTL}}^{\mathrm{sol}}\left(\tilde{n}, \tilde{\mathbf{t}}, \tilde{\mathbf{t}}^{*}\right)$ are defined respectively by (86) and by (65). Let $p_{m}$ in (65) be chosen by

$$
p_{m}= \pm \frac{a q^{m}+b}{b q^{m}+a}, \quad m \in S
$$

where $a=0$ or $b=0$, but $a b \neq 0$,(in particular, $p_{m}=q^{m}, m \in S$ ). Let the numbers $\xi_{m}$ in (86) be related to $\eta\left(\mathbf{t}_{o}, p_{m}\right)$ in (65) by

$$
\xi_{m}-\log \frac{(q ; q)_{m}}{(-q ; q)_{m}}=\eta_{m}:=2 \sum_{k=1}^{\infty}\left(p_{m}^{k}-p_{m}^{-k}\right)\left(\tilde{t}_{k}+\tilde{t}_{k}^{*}\right)+2 \tilde{n} \log p_{m}
$$


Then

$$
\tau_{1 \mathrm{DTL}}^{\mathrm{sol}}\left(\tilde{n}, \tilde{\mathbf{t}}+\tilde{\mathbf{t}}^{*}\right)=\tau\left(\mathbf{t}_{o}(q), \xi, \mathbf{t}_{o}(q)\right) .
$$

For the particular choice $p_{m}=m, m \in S$, this is also NLS multi-soliton tau function.

The hypergeometric function (92), where $x_{k}=q^{k-1}, k=1,2, \ldots$ and $N \rightarrow \infty$, is an example of multi-soliton 1DTL tau function evaluated at special values of times $\mathfrak{t}+\mathfrak{t}^{*}$, see (91), (107) and Remark 6.

REMARK 7. In case $S$ is a finite set, the polynomial $\tau$-function of type (85) (and (86)) is related to the soliton $\tau$-function with a finite number of solitons.

REMARK 8 . We note that the higher times $\mathbf{t}_{o}$ of the BKP hierarchy we started with are integrals of motion for (solitonic) $\tau$-function (72) of the second BTL hierarchy. Simultaneously, the higher times $\tilde{n}, \mathfrak{t}, \mathfrak{t}^{*}$ play the role of integrals of motion for the original BKP hierarchy. We therefore call these hierarchies dual to each other.

REMARK 9. An $\infty$-soliton solution with spectral parameters lying on a lattice appeared in [12-14] in a different way and in a different context. Other links between soliton and rational solutions of the KP hierarchy were found in [15].

4. Conclusions. An interesting problem is to study the asymptotic behaviour of hypergeometric $\tau$-functions. We hope to apply methods of soliton theory to conduct this study. We note that the asymptotic behaviour of infinite soliton $\tau$-functions, similar to those considered in the present paper, was studied in [13].

We hope to apply the series (1) to certain problems.

(1) Let us consider an integral

$$
\begin{aligned}
I\left(N, \mathbf{t}_{o}, \mathbf{t}_{o}^{*}\right)= & \frac{1}{N !} \iint_{\Gamma} \cdots \iint_{\Gamma} \prod_{i<j}^{N} \frac{\left(z_{i}-z_{j}\right)}{\left(z_{i}+z_{j}\right)} \frac{\left(z_{i}^{*}-z_{j}^{*}\right)}{\left(z_{i}^{*}+z_{j}^{*}\right)} \\
& \times \prod_{k=1}^{N} e^{\sum_{n=1,3, \ldots}^{\infty}\left(z_{k}^{n} t_{n}+z_{k}^{* n} t_{n}^{*}\right)} \mu\left(z_{k} z^{*}{ }_{k}\right) d z_{k} d z_{k}^{*},
\end{aligned}
$$

where $\Gamma$ is a integration domain in each $\left(z_{k}, z_{k}^{*}\right)$ plane $(k=1, \ldots, N)$, and $\mu$ is a function such that

$$
\iint_{\Gamma} \mu\left(z z^{*}\right) z^{n} d z d z^{*}=\iint_{\Gamma} \mu\left(z z^{*}\right) z^{* n} d z d z^{*}=\delta_{n, 0},
$$

and

$$
\iint_{\Gamma} \mu\left(z z^{*}\right) z^{n} z^{* m} d z d z^{*}=2 \delta_{n, m} e^{\xi_{n}} .
$$

The series (1) (in the case that the sum ranges over partitions of length $\ell(\lambda) \leq N$ ) yields the asymptotic expansion for the integral (109) [9]

$$
I\left(N, \mathbf{t}_{o}, \mathbf{t}_{o}^{*}\right)=\sum_{\lambda \in \mathrm{DP}, l(\lambda) \leq N} 2^{-l(\lambda)} e^{\sum_{i=1}^{\ell(\lambda)} \xi_{\lambda i}} Q_{\lambda}\left(\frac{1}{2} \mathbf{t}_{o}\right) Q_{\lambda}\left(\frac{1}{2} \mathbf{t}_{o}^{*}\right) .
$$

The restriction $\ell(\lambda) \leq N$ makes the difference between the r.h.s. of (112) and $\tau\left(\mathbf{t}_{o}, \xi, \mathbf{t}_{o}^{*}\right)$. 
In the limit $N \rightarrow \infty$ (which is typical for applications), the restriction $\ell(\lambda) \leq N$ is irrelevant for the perturbation series related to (109), and therefore, this series coincides with (1). Also, in the case that at least one of the sets $\mathbf{t}_{o}, \mathbf{t}_{o}^{*}$ has the form of

$$
m t_{m}=\sum_{k}^{N}\left(x_{k}^{m}-\left(-x_{k}\right)^{m}\right), \quad m t_{m}^{*}=\sum_{k}^{N}\left(y_{k}^{m}-\left(-y_{k}\right)^{m}\right),
$$

then by the bosonization formulae and Wick's Theorem we get that

$$
Q_{\lambda}\left(\frac{1}{2} \mathbf{t}_{o}\right)=0, \quad \ell(\lambda)>N
$$

Therefore, in this case, the integral $I\left(N, \mathbf{t}_{o}, \mathbf{t}_{o}^{*}\right)$ is the BKP $\tau$-function $\tau\left(\mathbf{t}_{o}, \xi, \mathbf{t}_{o}^{*}\right)$.

It may be interesting to apply (1) to matrix models and to statistical models where partition functions reduce to integrals (109), see, for instance, [11] for examples of integrals similar to (109).

It is interesting to compare integrals (109) with supersymmetric matrix models [33].

(2) The series (1) can be studied also in the context of random (strict) partitions.

Random strict partitions were considered in [16] and, in particular, the "shifted" measure $Q_{\lambda}(x) Q_{\lambda}(y)$ on (strict) partitions, were considered in [16]. In this paper, the series (1), where all $\xi_{n}=0$, and where $\lambda_{1}$ does not exceed a certain given number was studied.

Let us remark that the expression

$$
e^{\sum_{i=1}^{\infty} \xi_{\lambda_{i}}} Q_{\lambda}\left(\mathbf{t}_{\infty}\right) Q_{\lambda}\left(\mathbf{t}_{\infty}\right)=\prod_{i=1}^{\ell(\lambda)} e^{\xi_{i}}\left(\prod_{i=1}^{k} \frac{1}{\lambda_{i} !} \prod_{i<j}^{k} \frac{\lambda_{i}-\lambda_{j}}{\lambda_{i}+\lambda_{j}}\right)^{2}=\left(\frac{1}{H_{\lambda}^{*}}\right)^{2} e^{\sum_{i=1}^{\infty} \xi_{\lambda_{i}}}
$$

(where $H_{\lambda}^{*}$ is known to be related to the number of shifted tableau of the shape $\lambda$, see [17], [6]) in the case

$$
\xi_{n}=0, \quad n=0,1,2, \ldots
$$

may be considered as the analogue of the Plancherel measure [34], while in the case

$$
e^{\xi_{n}}=(z)_{n}(1-z)_{n}, \quad(z)_{n}=\frac{\Gamma(z+n)}{\Gamma(z)}, \quad n=0,1,2, \ldots
$$

as an analog of the so-called (z)-measure on random partitions (see [34]).

(3) Finally, we note that $\mathrm{KdV}$ soliton solutions with integer momenta was first considered in [12]. Each $\mathrm{KdV}$ solution of this type yields a wave operator $\partial_{t}^{2}-\Delta_{2 n-1}-u$, where the potential $u=2 \partial_{t_{1}}^{2} \tau_{\mathrm{KdV}}^{\text {sol }}\left(t_{1}\right)$, which satisfies the so-called generalized Huygens principle.

5. Appendix: Integral representations of scalar product $\langle,\rangle_{r}$, and of $\tau\left(\mathbf{t}, \xi, \mathbf{t}^{*}\right)[8]$. Consider a function $\mu$ of one variable with the property

$$
\iiint_{\Gamma} \mu\left(z z^{*}\right) z^{n} d z d z^{*}=\iint_{\Gamma} \mu\left(z z^{*}\right) z^{* n} d z d z^{*}=\delta_{n, 0}
$$


(As an example one can consider the case, when the variable $z^{*}$ is the complex conjugated to $z$, and $\Gamma$ is the whole complex plane).

Let us consider $\mu$ having only diagonal non-vanishing moments

$$
\iint_{\Gamma} \mu\left(z z^{*}\right) z^{n} z^{* m} d z d z^{*}=2 \delta_{n, m} e^{\xi_{n}}
$$

Examples:

(1) The variable $z^{*}$ is the complex conjugated to $z$, and $\Gamma$ is the whole complex plane. $\mu$ decays more rapidly then any power

(2) $\Gamma$ is a product of unit circles $\oint \oint, \mu$ is a Laurent series

(3) $\Gamma$ is a product of real and imaginary lines, $\mu\left(z z^{*}\right)=e^{z z^{*}}$

Using

$$
\begin{aligned}
& \frac{1}{M !} \int_{\Gamma} \cdots \int_{\Gamma} \phi\left(z_{M}\right) \cdots \phi\left(z_{1}\right)|0\rangle\langle 0| \phi\left(z_{1}^{*}\right) \cdots \phi\left(z_{M}^{*}\right) \prod_{i=1}^{M} \mu_{r}\left(z_{i} z_{i}^{*}\right) d z_{i} d z_{i}^{*} \\
& \quad=\sum_{\lambda \in \mathrm{DP}, \ell(\lambda) \leq M} 2^{\ell(\lambda)}|\lambda\rangle r_{\lambda}\langle\lambda|,
\end{aligned}
$$

where for $\lambda=\left(\lambda_{1}, \ldots, \lambda_{k}\right)$

$$
|\lambda\rangle=\phi_{\lambda_{1}} \cdots \phi_{\lambda_{k}}|0\rangle
$$

we obtain for partitions $\lambda, \mu$ (both partitions have length $\ell(\lambda), \ell(\mu) \leq M$ )

$$
\frac{1}{M !} \int \cdots \int \Delta(z) \Delta\left(z^{*}\right) Q_{\lambda}(z) Q_{\mu}\left(z^{*}\right) \prod_{k=1}^{M} \mu_{r}\left(z_{k} z^{*}\right) d z_{k} d z_{k}^{*}=2^{l(\lambda)} r_{\lambda} \delta_{\lambda \mu},
$$

where

$$
\Delta(z)=\prod_{i<j}^{M} \frac{\left(z_{i}-z_{j}\right)}{\left(z_{i}+z_{j}\right)}, \quad \Delta\left(z^{*}\right)=\prod_{i<j}^{M} \frac{\left(z_{i}^{*}-z_{j}^{*}\right)}{\left(z_{i}^{*}+z_{j}^{*}\right)}
$$

The relation (122) yields the integral representation for the inner product

$$
\left\langle Q_{\mu}, Q_{\lambda}\right\rangle_{r}=2^{\ell(\lambda)} r_{\lambda} \delta_{\mu, \lambda},
$$

With the help of equalities

$$
\begin{aligned}
e^{\sum_{n=1,3, \ldots}^{\infty} \sum_{k=1}^{M} z_{k}^{n} t_{n}} & =\sum_{\lambda \in \mathrm{DP}, l(\lambda) \leq M} 2^{-\ell(\lambda)} Q_{\lambda}\left(\mathbf{z}^{M}\right) Q_{\lambda}\left(\mathbf{t}_{o}\right), \\
e^{\sum_{n=1,3, \ldots}^{\infty} \sum_{k=1}^{M} z_{k}^{* n} t_{n}^{*}} & =\sum_{\lambda \in \mathrm{DP}, \ell(\lambda) \leq M} 2^{-l(\lambda)} Q_{\lambda}\left(\mathbf{z}^{* M}\right) Q_{\lambda}\left(\mathbf{t}_{o}^{*}\right)
\end{aligned}
$$

we evaluate the integral

$$
I_{r}\left(M, \mathbf{t}_{o}, \mathbf{t}_{o}^{*}\right)=\frac{1}{M !} \int \cdots \int \Delta(z) \Delta\left(z^{*}\right) \prod_{k=1}^{M} e^{\sum_{n=1,3, \ldots}^{\infty}\left(z_{k}^{n} t_{n}+z_{k}^{* n} t_{n}^{*}\right)} \mu_{r}\left(z_{k} z_{k}^{*}\right) d z_{k} d z_{k}^{*}
$$


We finally obtain

$$
I_{r}\left(M, \mathbf{t}_{o}, \mathbf{t}_{o}^{*}\right)=\sum_{\lambda \in \mathrm{DP}, l(\lambda) \leq M} 2^{-l(\lambda)} r_{\lambda} Q_{\lambda}\left(\frac{\mathbf{t}_{o}}{2}\right) Q_{\lambda}\left(\frac{\mathbf{t}_{o}^{*}}{2}\right)
$$

The restriction $\ell(\lambda) \leq M$ causes the difference between the right-hand side of (128) and $\tau_{r}\left(\mathbf{t}, \xi, \mathbf{t}^{*}\right)$.

However, in case at least one of the sets $\mathbf{t}_{o}, \mathbf{t}_{o}^{*}$ has the form of

$$
m t_{m}=\sum_{k}^{N_{1}}\left(x_{k}^{m}-\left(-x_{k}\right)^{m}\right), \quad m t_{m}^{*}=\sum_{k}^{N_{2}}\left(y_{k}^{m}-\left(-y_{k}\right)^{m}\right)
$$

with $N$ or $N^{\prime}$ no more then $M$, then

$$
Q_{\lambda}\left(\frac{1}{2} \mathbf{t}_{o}\right)=0, \quad \ell(\lambda)>M
$$

Therefore, in this case, the integral $I_{r}\left(M, \mathbf{t}_{o}, \mathbf{t}_{o}^{*}\right)$ is the BKP $\tau$-function $\tau_{r}\left(\mathbf{t}_{o}, \mathbf{t}_{o}^{*}\right)$.

ACKNOWLEDGEMENTS. The authors are grateful to T. Shiota for useful discussion. The work was supported in part by the Russian Foundation for Fundamental Researches (Grant No 02-02-17382), and the Program of Russian Academy of Science "Mathematical Methods in Nonlinear Dynamics". We thank Claire Gilson and Chris Athorne for the organization of the visit of one of the authors (A.O.) to the University of Glasgow, during which work on this paper was initiated.

\section{REFERENCES}

1. E. Date, M. Jimbo, M. Kashiwara and T. Miwa, Transformation groups for soliton equations, in Nonlinear integrable systems - classical theory and quantum theory, eds. M. Jimbo and T. Miwa (World Scientific, 1983), 39-120.

2. E. Date, M. Jimbo, M. Kashiwara and T. Miwa, Physica 4D (1982), 343.

3. Y. You, Polynomial solutions of the BKP hierarchy and projective representations of symmetric groups, in Infinite-dimensional Lie algebras and groups, Adv. Ser. Math. Phys. 7 (1990), $449-464$.

4. J. J. C. Nimmo, J. Physics A 23 (1990), 751.

5. I. Schur, J.Reine Angew. Math. 139 (1911), 155. 1995).

6. I. G. Macdonald, Symmetric functions and Hall polynomials (Clarendon Press, Oxford,

7. V. E. Zakharov and A. B. Shabat, J. Funct. Anal. Appl. 8 (1974), 226.

8. A. Yu. Orlov, Hypergeometric $\tau$-functions $\tau\left(\mathbf{t}, T, \mathbf{t}^{*}\right)$ as $\infty$-soliton $\tau$-function in $T$ variables, nonlin.SI/0305001.

9. A. Yu. Orlov, Theoretical and Mathematical Physics 137 (2003), 1573-1588.

10. A. Yu. Orlov, Soliton theory, symmetric functions and matrix integrals, SI/0207030 v1 Acta Applicandae Mathematicae, 2004, to appear.

11. I. K. Kostov, Solvable statistical models on a random lattice hep-th/9509124 v4.

12. Yu. Yu. Berest and I. M. Loutsenko, Comm. Math. Phys. 190 (1997), 113.

13. I. M. Loutsenko and V. Spiridonov, Journal of Statistical Physics 99 (2000), 751.

14. I. Loutsenko and V. Spiridonov, Self-similarity in spectral problems and q-special functions, Nuclear Phys. B 538 (1998), 731, solv-int/9909022.

15. A. Mironov, Seiberg-Witten theory and duality in integrable systems, hep-th/0011093.

16. C. Tracy and H. Widom, A limit theorem for shifted schur measur math.PR/0210255.

17. J. R. Stembridge, J. Alg. Combinatorics 1 (1992), 71-95. 
18. K. I. Gross and D. S. Richards: Trans. Amer. Math. Soc. 301 (1987), 781.

19. S. C. Milne, Summation theorems for basic hypergeometric series of Schur function argument, in Progress in Approximation Theory (Springer-Verlag, 1992), 51-77.

20. N. A. Nekrasov, Seiberg-Witten prepotential from instanton counting, hep-th/0206161 $v 1$.

21. A. S. Losev, A. Marshakov and N. A. Nekrasov, Small instantons, little strings and free fermions, hep-th/0302191 v3.

22. A. Okounkov, Toda equations for Hurwitz numbers, $A G / 00044128 v 1$.

23. A. Okounkov and R. Pandharipande, The equivariant Gromov-Witten theory on $P^{1}$, $A G / 0207233$ v1.

24. Wei-Ping Li, Zhenbo Qin and Weiqiang Wang, Hilbert schemes, integrable hierarchies and Gromov-Witten theory, $A G / 0302211 v 1$.

25. A. Migdal, Zh. Eksp. Teor. Fiz. 69 (1975), 810.

26. K. Saaidi and M. Khorrami, Large $N$ limit of the nonlocal 2D Yang-Mills and generalized Yang-Mills theories on a cylinder, hep-th/0304204 vl. 1690.

27. J. Harnad and A. Yu. Orlov, Theoretical and Mathematical Physics 137 (2003), 1676-

28. A. Yu. Orlov, New Solvable Matrix Integrals, nlin. SI/0209063 Int. J. Math. Phys. A (2004), to appear.

29. A. Yu. Orlov, New Solvable Matrix Models-U(n) case, in, Nonlinear Physics: Theory and Experiment. II, Universita' di Lecce - Consortium Einstein; editors: M. J. Ablowitz, M. Boiti, F. Pempinelli, B. Prinari (World Scientific, 2002), 99-100.

30. T. Nakatsu, K. Takasaki and S. Tsujimaru, Nucl. Phys. B 443 (1995), 155.

31. K. Takasaki, Comm. Math. Phys. 181 (1996), 131.

32. K. Ueno and K. Takasaki, Adv. Stud. Pure Math. 4 (1984), 1-95.

33. T. Guhr, J. Math. Phys. 32(2), February 1991.

34. A. Borodin and G. Olshanski, Random partitions and Gamma kernel, mathph/0305043. 